\title{
Effectiveness of afatinib in an NSCLC patient with EGFR mutation and early progression to osimertinib: a case report
}

\author{
Koichiro Nozaki $^{1 \wedge}$, Satoshi Watanabe ${ }^{1 \wedge}$, Kazuto Nishio $^{2}$, Kazuko Sakai $^{2}$, Toshiaki Kikuchi ${ }^{1}$ \\ ${ }^{1}$ Department of Respiratory Medicine and Infectious Diseases, Niigata University Graduate School of Medical and Dental Sciences, Niigata City, \\ Niigata, Japan; ${ }^{2}$ Department of Genome Biology, Kindai University Faculty of Medicine, Osaka-Sayama City, Osaka, Japan \\ Correspondence to: Satoshi Watanabe. Department of Respiratory Medicine and Infectious Diseases, Niigata University Graduate School of Medical \\ and Dental Sciences, 1-754, Asahimachi-dori, Niigata, 951-8520, Japan. Email: satoshi7@med.niigata-u.ac.jp.
}

\begin{abstract}
Osimertinib, a third-generation epidermal growth factor receptor (EGFR) tyrosine kinase inhibitor (TKI), shows great clinical activity in non-small cell lung cancer (NSCLC) patients with EGFR mutations regardless of T790M mutation at first-line chemotherapy. Previous studies demonstrated that there are few patients with initial resistance to osimertinib. Here, we describe a case to report the efficacy of afatinib in an EGFR-mutated NSCLC patient with early progression to first-line osimertinib treatment. A 68-year-old Japanese male was diagnosed with stage IVB lung adenocarcinoma with the EGFR L858R mutation in exon 21. Two months after the start of osimertinib, his tumor progressed at the initial response evaluation. Because he refused to receive cytotoxic chemotherapy, afatinib treatment was initiated. He was administered afatinib, and the tumor shrank. After five months of afatinib treatment, nevertheless the primary tumor was not enlarged, he experienced disease progression with leptomeningeal metastasis and passed away. To elucidate the resistance mechanisms of osimertinib in this patient, we performed nextgeneration sequencing (NGS) on tumor samples from pleural effusions after osimertinib failure. NGS revealed no specific gene mutations causing resistance to osimertinib except for the EGFR L858R mutation; however, his tumor had a relatively high tumor mutational burden. Afatinib is considered an option for EGFR-mutated patients with early progression to osimertinib.
\end{abstract}

Keywords: Epidermal growth factor receptor (EGFR); non-small cell lung cancer (NSCLC); afatinib; early progression to osimertinib; case report

Submitted Sep 09, 2021. Accepted for publication Dec 13, 2021.

doi: $10.21037 /$ tcr-21-1850

View this article at: https://dx.doi.org/10.21037/tcr-21-1850

\section{Introduction}

Osimertinib, a third-generation epidermal growth factor receptor (EGFR) tyrosine kinase inhibitor (TKI), is effective in cases where T790M is detected after resistance to firstor second-generation EGFR-TKIs and shows great clinical activity in non-small cell lung cancer (NSCLC) patients with EGFR mutations regardless of T790M mutation at first-line chemotherapy $(1,2)$. Afatinib, second-generation EGFR-TKI, was reported to be effective for NSCLC with uncommon EGFR mutations and compound EGFR mutations (3). However, there is no report that Afatinib is effective in EGFR mutation-positive lung cancer without uncommon and compound EGFR mutations that increases early after treatment with osimertinib.

Here, we report a case of EGFR-mutated NSCLC treated with afatinib after early progression to osimertinib. We present the following article in accordance with the CARE reporting checklist (available at https://tcr.amegroups.com/ article/view/10.21037/tcr-21-1850/rc).

\footnotetext{
^ ORCID: Koichiro Nozaki, 0000-0003-0215-2393; Satoshi Watanabe, 0000-0003-0041-6981.
} 

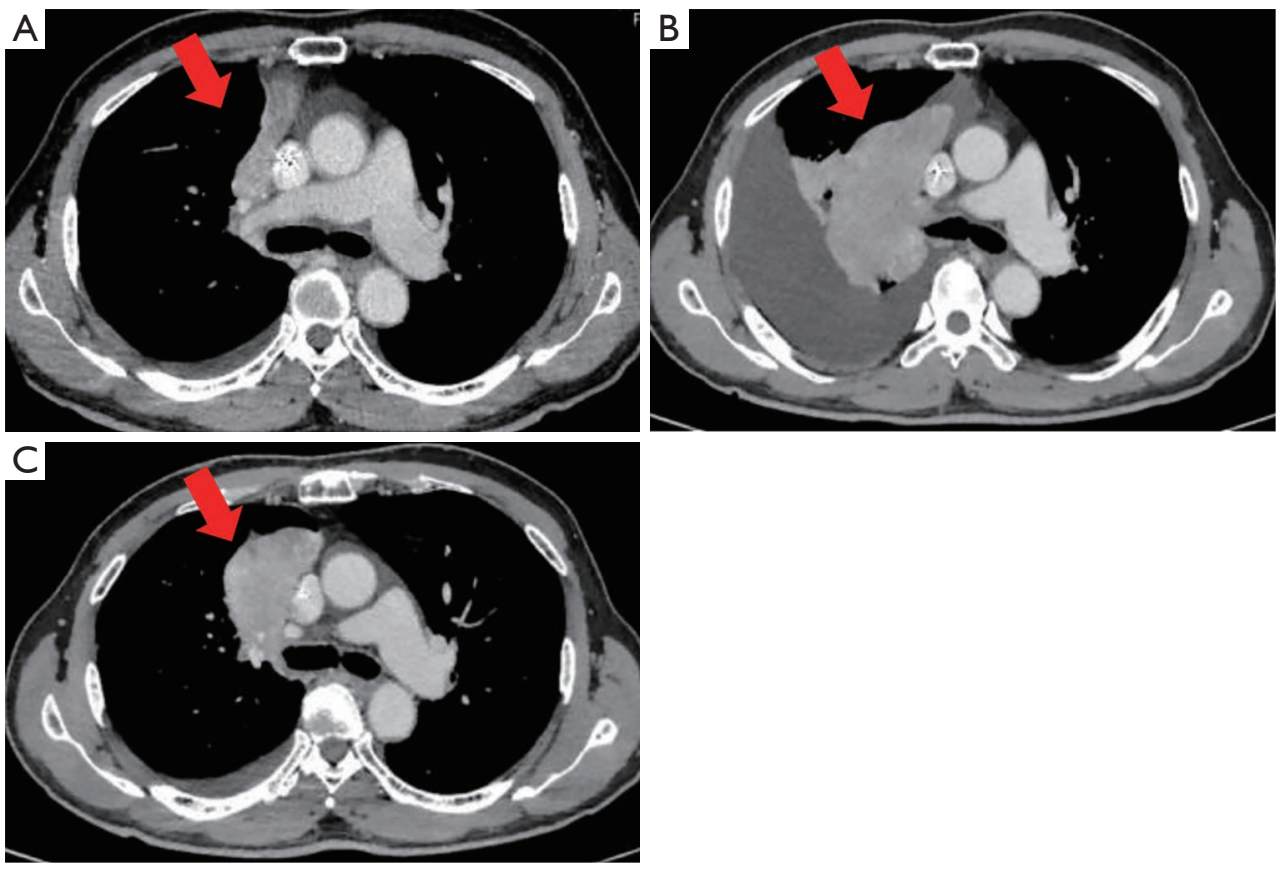

Figure 1 Chest CT images before and after afatinib treatment. The CT showed a primary tumor (red arrow) in the upper lobe of the right lung before osimertinib treatment (A). Two months after osimertinib treatment, rapid progression of primary tumor (red arrow) and pleural effusion were revealed by the CT (B). After one month of afatinib treatment, the CT demonstrated a good response of primary tumor (red arrow) and pleural effusion (C). CT, computed tomography.

\section{Case presentation}

A 68-year-old Japanese male with a smoking history was diagnosed with stage IVB lung adenocarcinoma with multiple bone metastases (cT3N1M1c, Figure 1A). EGFR gene detection showed the L858R mutation in exon 21 . The patient started osimertinib treatment as a first-line chemotherapy. Two months after the start of osimertinib, a computed tomography (CT) scan revealed tumor progression (Figure 1B). We confirmed that this patient had no financial problems and was taking osimertinib daily. Because he refused to receive cytotoxic chemotherapy, afatinib treatment was initiated. After a month of treatment with afatinib, a CT scan showed a good response of the primary tumor in the upper right lobe (Figure 1C). To elucidate the resistance mechanisms of osimertinib in this patient, we performed amplicon sequencing (Ion AmpliSeq Comprehensive Cancer Panel, ThermoFisher Scientific, Waltham, MA) on tumor samples from pleural effusions after osimertinib failure. Amplicon sequencing revealed a high tumor mutational burden (TMB) (8.56 mutations per $\mathrm{Mb}$ ); however, we found neither compound $E G F R$ mutations nor HER-family gene mutations except for the EGFR L858R mutation. After five months of afatinib treatment, nevertheless the primary tumor was not enlarged, he experienced disease progression with leptomeningeal metastasis and passed away.

All procedures performed in this study involving human participants were in accordance with the ethical standards of the institutional and/or national research committee(s) and with the Helsinki Declaration (as revised in 2013). Written informed consent was obtained from the patient for publication of this case report and accompanying images. A copy of the written consent is available for review by the editorial office of this journal.

\section{Discussion}

Previous studies have demonstrated that there are few patients who have early progression to first-line osimertinib treatment. In the phase III FLAURA trial, first-line osimertinib had great treatment responses in EGFRmutated NSCLC patients, and only $1 \%$ of patients ( 3 of 279) showed progressive disease as the best response in the osimertinib arm (2). Although the mechanisms of acquired 
resistance to first-line osimertinib have been reported [MET amplification (15\%), C797X (7\%), PIK3CA mutation (7\%), KRAS mutation (3\%) and HER2 amplification (2\%)] (4), c-Myc modulation (5), CD74 upregulation (6), and structural changes of EGFR mutations (7), the mechanisms of intrinsic resistance to osimertinib are largely unknown. Similarly, next-generation sequencing (NGS) analysis of tumor samples from our patient failed to find specific gene mutations or amplifications that caused resistance to osimertinib.

Afatinib was reported to be effective for NSCLC with uncommon EGFR mutations and compound EGFR mutations (3). In addition, there have been NSCLC patients with compound EGFR mutations who respond to afatinib immediately after osimertinib failure $(8,9)$. Although we expected that tumor cells from our patient could have compound EGFR mutations and although the tumor showed a response to afatinib, NGS analysis did not demonstrate any uncommon or compound EGFR mutations in our patient. A phase I study investigating the safety of the combination of afatinib and osimertinib after failure of osimertinib reported a response rate of $7.7 \%$, suggesting that the efficacy of afatinib combination therapy may be limited, but no predictive biomarkers are known (10). Previous studies observed a higher TMB in patients harboring EGFR L858R mutations than in those carrying exon 19 deletions, and a high TMB was correlated with a shorter survival time in patients with EGFR-mutated NSCLC receiving EGFR-TKIs (11). There is also a report that afatinib treatment decreased TMB (12). These findings indicate that afatinib is more effective for patients with high TMB EGFR-mutated NSCLC than other EGFR-TKIs. Because our patient had EGFR-mutated NSCLC with a high TMB, this could be a reason why afatinib had clinical activity in our patient after resistance to osimertinib. The limitation of this case was that the rebiopsy specimen was a cell block of pleural fluid, not from an enlarging primary tumor. In addition, if liquid biopsy had been performed, we may have found a specific gene mutation involved in EGFR-TKI resistance.

To the best of our knowledge, this is the first case to report the effectiveness of afatinib for EGFR-mutated NSCLC with early progression to osimertinib. Our findings suggest that the administration of afatinib is a treatment option in EGFR-mutated NSCLC patients with initial tolerance to first-line osimertinib.

\section{Acknowledgments}

Funding: None.

\section{Footnote}

Reporting Checklist: The authors have completed the CARE reporting checklist. Available at https://tcr.amegroups.com/ article/view/10.21037/tcr-21-1850/rc

Peer Review File: Available at https://tcr.amegroups.com/ article/view/10.21037/tcr-21-1850/prf

Conflicts of Interest: All authors have completed the ICMJE uniform disclosure form (Available at https://tcr.amegroups. com/article/view/10.21037/tcr-21-1850/coif). K Nozaki reports that the author receives honoraria for lectures from AstraZeneca, Boehringer Ingelheim, Taiho Pharmaceutical and MSD. Satoshi Watanabe reports that the author receives honoraria for lectures from AstraZeneca, Chugai Pharma, Ono Pharmaceutical, Bristol-Myers, Boehringer Ingelheim, Eli Lilly, MSD, Taiho Pharmaceutical, Pfizer, Novartis and Daiichi Sankyo. K Nishio reports that the author received lecture fees from CHUGAI PHARMACEUTICAL Co., Ltd., Eisai Co., Ltd., Pfizer Inc., Nippon Boehringer Ingelheim Co., Ltd., Novartis Pharma K.K., MSD K.K., ONO PHARMACEUTICAL Co., Ltd., Bristol-Myers Squibb Company, SymBio Pharmaceuticals Ltd., Life Technologies Japan Ltd., Solasia Pharma K.K., Eli Lilly Japan K.K., Yakult Honsha Co., Ltd., Roche Diagnostics K.K., Life Technologies Japan Ltd., AstraZeneca K.K., Otsuka Pharmaceutical Co., Ltd., Sanofi K.K., Guardant Health Inc. KS reports that the author receives honoraria for lectures from Roche Diagnostics, Bio-Rad, AstraZeneca, Chugai Pharmaceutical, and Hitachi. Toshiaki Kikuchi reports that the author receives grants from Nippon Boehringer Ingelheim Co., Ltd., and AstraZeneca K.K., and honoraria for lectures from Nippon Boehringer Ingelheim Co., Ltd., and AstraZeneca K.K. The authors have no other conflicts of interest to declare.

Ethical Statement: The authors are accountable for all aspects of the work in ensuring that questions related to the accuracy or integrity of any part of the work are appropriately investigated and resolved. All procedures performed in this study involving human participants were in accordance with the ethical standards of the institutional and/or national research committee(s) and with the Helsinki Declaration (as revised in 2013). Written informed consent was obtained from the patient for publication of this case report and accompanying images. A copy of the written consent is available for review by the editorial office of this journal. 
Open Access Statement: This is an Open Access article distributed in accordance with the Creative Commons Attribution-NonCommercial-NoDerivs 4.0 International License (CC BY-NC-ND 4.0), which permits the noncommercial replication and distribution of the article with the strict proviso that no changes or edits are made and the original work is properly cited (including links to both the formal publication through the relevant DOI and the license). See: https://creativecommons.org/licenses/by-nc-nd/4.0/.

\section{References}

1. Mok TS, Wu YL, Ahn MJ, et al. Osimertinib or PlatinumPemetrexed in EGFR T790M-Positive Lung Cancer. N Engl J Med 2017;376:629-40.

2. Soria JC, Ohe Y, Vansteenkiste J, et al. Osimertinib in Untreated EGFR-Mutated Advanced Non-Small-Cell Lung Cancer. N Engl J Med 2018;378:113-25.

3. Yang JC, Schuler M, Popat S, et al. Afatinib for the Treatment of NSCLC Harboring Uncommon EGFR Mutations: A Database of 693 Cases. J Thorac Oncol 2020;15:803-15.

4. Ramalingam SS, Cheng Y, Zhou C, et al. LBA50: mechanisms of acquired resistance to first-line osimertinib:preliminary data from the phase III FLAURA study. Ann Oncol 2018;29:viii740.

5. Zhu L, Chen Z, Zang H, et al. Targeting c-Myc to Overcome Acquired Resistance of EGFR Mutant NSCLC Cells to the Third-Generation EGFR Tyrosine Kinase

Cite this article as: Nozaki K, Watanabe S, Nishio K, Sakai K, Kikuchi T. Effectiveness of afatinib in an NSCLC patient with EGFR mutation and early progression to osimertinib: a case report. Transl Cancer Res 2022;11(1):295-298. doi: 10.21037/ tcr-21-1850
Inhibitor, Osimertinib. Cancer Res 2021;81:4822-34.

6. Kashima Y, Shibahara D, Suzuki A, et al. Single-Cell Analyses Reveal Diverse Mechanisms of Resistance to EGFR Tyrosine Kinase Inhibitors in Lung Cancer. Cancer Res 2021;81:4835-48.

7. Robichaux JP, Le X, Vijayan RSK, et al. Structure-based classification predicts drug response in EGFR-mutant NSCLC. Nature 2021;597:732-7.

8. Fang W, Gan J, Huang Y, et al. Acquired EGFR L718V Mutation and Loss of T790M-Mediated Resistance to Osimertinib in a Patient With NSCLC Who Responded to Afatinib. J Thorac Oncol 2019;14:e274-5.

9. Liu Y, Li Y, Ou Q, et al. Acquired EGFR L718V mutation mediates resistance to osimertinib in non-small cell lung cancer but retains sensitivity to afatinib. Lung Cancer 2018;118:1-5.

10. Miura S, Azuma K, Yoshioka H, et al. MA02.05: A Phase I Study of Afatinib in Combination With Osimertinib in Patients After Failure of Prior Osimertinib. J Thorac Oncol 2021;16:S890-1.

11. Offin M, Rizvi H, Tenet M, et al. Tumor Mutation Burden and Efficacy of EGFR-Tyrosine Kinase Inhibitors in Patients with EGFR-Mutant Lung Cancers. Clin Cancer Res 2019;25:1063-9.

12. Ishii H, Azuma K, Sakai K, et al. Determination of Somatic Mutations and Tumor Mutation Burden in Plasma by CAPP-Seq during Afatinib Treatment in NSCLC Patients Resistance to Osimertinib. Sci Rep 2020;10:691. 